\title{
Diagnostic difficulties in neuroendocrine tumors - intrapancreatic accessory spleen
}

Anna Skowrońska1 , Tomasz Bednarczuk¹, Joanna Podgórska², Dorota Kaczmarska-Turek ${ }^{1}$, Jarosław B. Ćwikła ${ }^{3}$

'Department of Internal Medicine and Endocrinology, Medical University of Warsaw, Poland

2Second Department of Clinical Radiology, Meadical University of Warsaw, Poland

${ }^{3}$ Faculty of Medical Sciences, University of Warmia and Mazury, Olsztyn, Poland

[Received 1 XII 2016; Accepted 29 III 2017]

Accessory spleens are congenital abnormalities, being identified in approximately $7-15 \%$ of population. Their most frequent location is splenic hilum, pancreatic tail, gastro-splenic ligament, intestinal wall, mesentery, greater omentum or uterine appendages.

Intrapancreatic accessory spleens (IPAS) usually pose no clinical problem. With the development of improved imaging techniques, IPAS are more readily detected. The occasional findings may mimic other types of a mass-forming lesion in the tail of the pancreas, particular pancreatic neuroendocrine neoplasms (pNEN), adenocarcinoma, pseudopapillary tumors and metastases.

We report a case of 40-year-old woman with history of gastroesophageal reflux disease admitted to the Clinic of Endocrinology with a suspicion of serotonin-secreting neuroendocrine neoplasm. She reported an atypical symptoms of carcinoid syndrome such as sporadic but intensive flushing, fatigue and weight loss of approximately 4-5 kg for 5 years. Her family history was unremarkable. She reported the use of proton pomp inhibitor esomeprazole $40 \mathrm{mg}$ daily.

A physical examination and additional laboratory data findings were unremarkable. Level of serum chromogranin A was

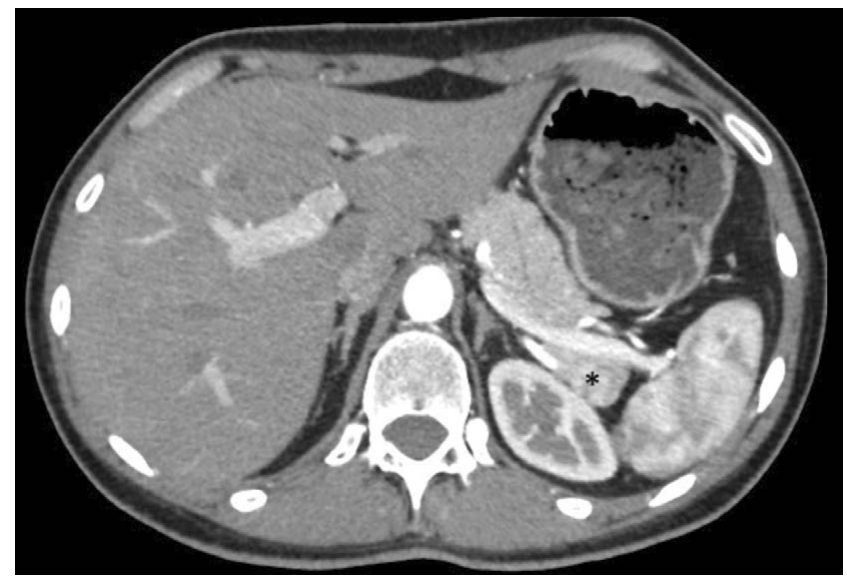

Figure 1.

Correspondence to: A. Skowrońska

Department of Internal Medicine and Endocrinology

Medical University of Warsaw

Banacha 1a, 02-097 Warsaw, Poland

Tel: +48225992975 elevated (346 $\mu \mathrm{g} / \mathrm{dL}$; normal: 0-94 $\mu \mathrm{g} / \mathrm{dL}$ ), however normalized after cessation of proton pomp inhibitor therapy. The concentration of 5-hydroxyindoloacetic acid (5-HIAA) in 24-hour urine collection was in normal range.

Abdomen computed tomography (CT) scans showed round homogenous lesion of $20 \mathrm{~mm}$ in diameter located in the distal part of the tail of the pancreas with the intensive arterial enhancement (Figure 1). In further diagnostic procedure, magnetic resonance imaging (MRI) of the abdomen was performed and revealed a $17 \mathrm{~mm}$ well-defined mass with spleen-like low signal intensity on T1 (Figure 2A), T2-hyperintense fat saturated images (Figure 2B) and significant heterogeneous contrast enhancement in arterial phase, followed by
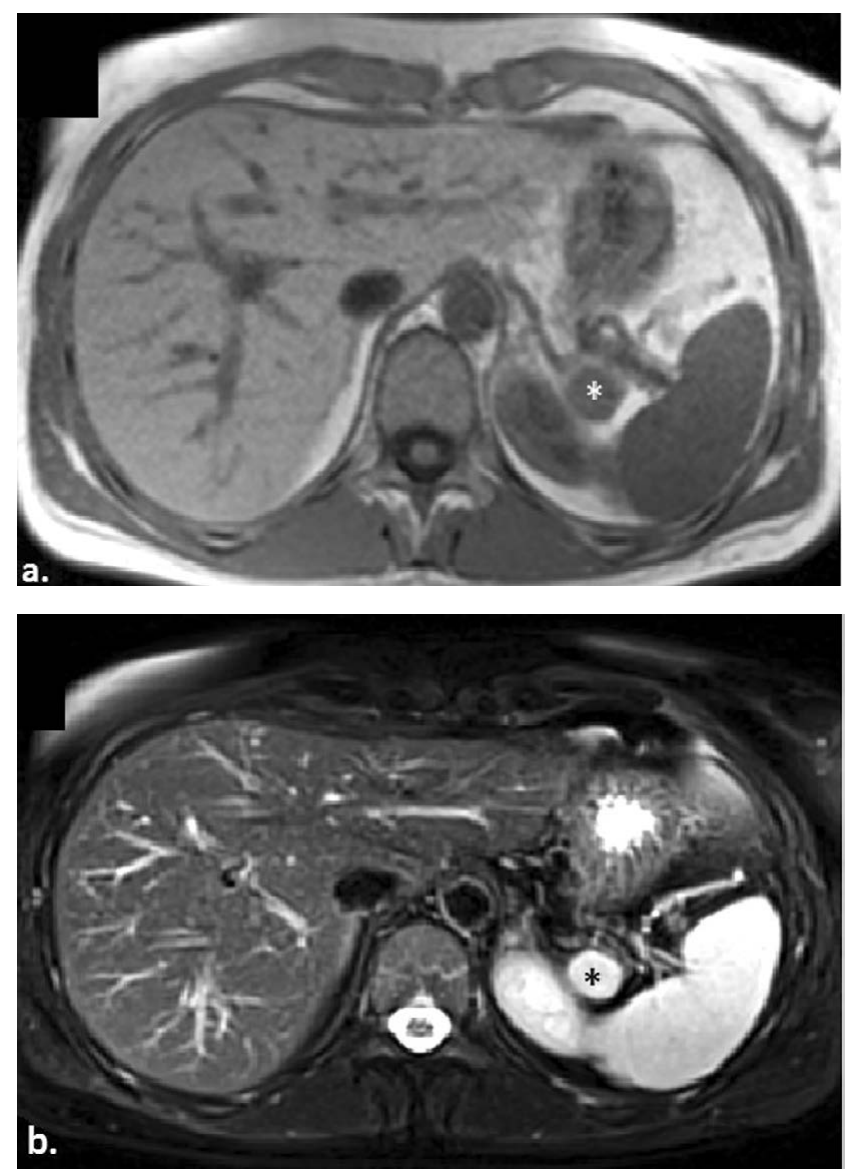

Figure 2. 


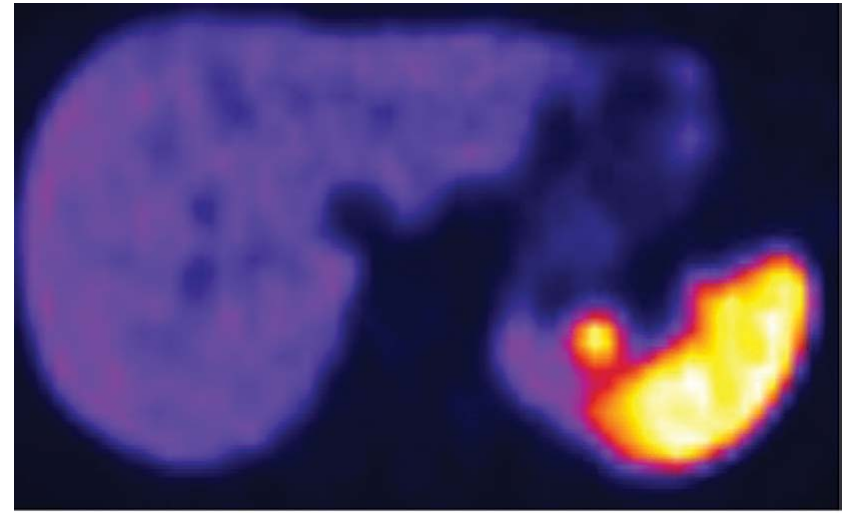

Figure 3.

homogeneous enhancement in venous and delayed phases. Based on radiographic findings, pNEN, IPAS or pseudopapillary tumor were suspected. Gallium-68 ( ${ }^{68} \mathrm{Ga}$-DOTA-TATE) PET/CT revealed a mass in the tail of pancreas with intensive uptake of the tracer (Figure 3). Endoscopic ultrasonography (EUS) was performed and demonstrated the oval shaped, well-defined, and homogeneous, hypoechoic lesion in the tail of the pancreas, which presented similar echogenicity to the spleen. A cytological result of fine needle aspiration (FNA) biopsy showed large aggregates of plasma cells leukocyte count, with no epithelium cell and the cell block were immunonegative for synaptophysin and chromogranin.

Since the etiology of the mass in the tail of the pancreas remains unclear, technetium-99m Single-photon emission computed tomography (SPECT) using 99mTc-labelled tin colloid (PoltechColloid, NCBJ, Polatom, PL), was performed to differentiate spleen tissue from neuroendocrine tumour. The high uptake of radiotracer in suspicious mass in the pancreatic distal tail close to spleen hilum was consistent with accessory spleen (Figure 4).

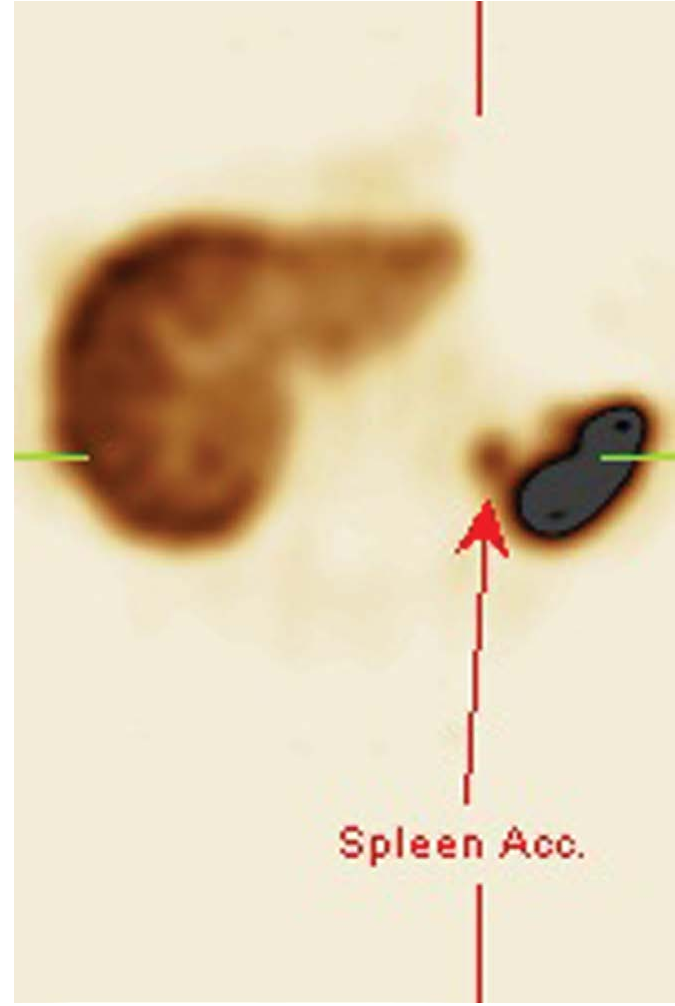

Figure 4.

Considering the patient's diagnostic process, we suggest that clinical and radiological approach is essential for making the correct diagnosis of IPAS leading to a good prognosis and prevention of unnecessary medical procedures. 